Research Paper

\title{
Cytological Kinetics of Periodontal Ligament in an Experimental Occlusal Trauma Model
}

\author{
Tatsuo Takaya ${ }^{\circledR}$, Hiroaki Mimura ${ }^{1}$, Saeka Matsuda², Keisuke Nakano², Hidetsugu Tsujigiwa², Mihoko \\ Tomida ${ }^{4}$, Norimasa Okafuji ${ }^{2}$, Takeo Fujiii ${ }^{1}$, and Toshiyuki Kawakami² \\ 1. Department of Oral Health Promotion, Matsumoto Dental University Graduate School of Oral Medicine, Shiojiri, Japan \\ 2. Department of Hard Tissue Research, Matsumoto Dental University Graduate School of Oral Medicine, Shiojiri, Japan \\ 3. Department of Life Science, Faculty of Science, Okayama University of Science, Okayama, Japan \\ 4. Department of Oral and Maxillofacial Biology, Matsumoto Dental University School of Dentistry, Shiojiri, Japan
}

$\bowtie$ Corresponding author: tachy@po.mdu.ac.jp

() 2015 Ivyspring International Publisher. Reproduction is permitted for personal, noncommercial use, provided that the article is in whole, unmodified, and properly cited. See http:/ /ivyspring.com/terms for terms and conditions.

Received: 2015.03.23; Accepted: 2015.06.01; Published: 2015.06.23

\begin{abstract}
Using a model of experimental occlusal trauma in mice, we investigated cytological kinetics of periodontal ligament by means of histopathological, immunohistochemical, and photographical analysis methods. Periodontal ligament cells at furcation areas of molar teeth in the experimental group on day 4 showed a proliferation tendency of periodontal ligament cells. The cells with a round-shaped nucleus deeply stained the hematoxylin and increased within the day 4 specimens. Ki67 positive nuclei showed a prominent increase in the group on days 4 and 7 . Green Fluorescent Protein (GFP) positivity also revealed cell movement but was slightly slow compared to Ki67. It indicated that restoration of mechanism seemed conspicuous by osteoclasts and macrophages from bone-marrow-derived cells for the periodontal ligament at the furcation area. It was suggested that the remodeling of periodontal ligament with cell acceleration was evoked from the experiment for the group on day 4 and after day 7 . Periodontal ligament at the furcation area of the molar teeth in this experimental model recovered using the cells in situ and the bone-marrow-derived cells.
\end{abstract}

Key words: Occlusal trauma, Periodontal tissue, Green fluorescent protein (GFP), Ki67, Mouse

\section{Introduction}

Occlusal trauma is defined as an injury resulting in tissue changes within the attachment apparatus as a result of occlusal forces. It has been proved in many studies that occlusal trauma can cause a variety of destructive biological effects on periodontal tissues (1-4). It has been suggested that occlusal trauma causes various destructive effects on the periodontal tissue, and two theories have been proposed relating to occlusal trauma: one is the "co-destructive factor theory" by Glickman (5), which suggests inflammatory changes induced by infection with periodontal pathogens and occlusal trauma caused by excessive occlusal loading both greatly contribute to the progression of periodontal diseases, especially those characterized by a large amount of alveolar bone re- sorption; and the other is the theory proposed by Waerhaug (6), which denies occlusal trauma as a co-factor in the loss of connective tissue attachment and vertical alveolar bone resorption. More recently, studies on animal models have examined research concerning occlusal trauma using monkeys (12). The experiment combined different levels of inflammation and different types of trauma. Specifically, the authors ligated the molars of monkeys using a cotton thread to induce severe inflammation, and then the monkeys were divided into two groups: one received traumatic force and the other nothing. The resulting histopathological reports indicated the more severe the periodontal tissue inflammation, the greater the tissue destruction that resulted from the traumatic occlu- 
sion, and that more severe tissue destruction occurred when traumatic force was applied in two directions, rather than one (12). Experimental mechanical stress causes changes in periodontal tissues. This has been reported by previous study (13) from an orthodontic point of view. According to the results, the changes in the pressured side of periodontal tissues were severe. However, if the cytotoxic stress is in short term, the mechanism for repairing the periodontal ligaments could be observed (13). There are still many unknowns because experimental validations have been performed mostly from a histopathological standpoint, and experimental validation of the cellular kinetics of periodontal tissues in an in vivo experimental system has not made progress (7-12). These research reports failed to performed sufficient validation on the cytological kinetics of the periodontal tissues in the case of occlusal trauma. In the view of establishing an animal experimental system that is highly versatile and repeatable, we built an experimental system in which overload is added to the molar region of mice, and we reexamined the periodontal tissues from the viewpoint of cytological kinetics (23). We then performed histopathological and also immunohistochemical examinations.

\section{Materials and Methods}

\section{1) Experimental animals}

Eleven 7-week-old ddY male mice (weighing 35 \pm 2 g) (Japan SLC Inc., Hamamatsu, Japan) and eight 7-week-old bone marrow transplanted female C57BL/ 6 genealogy mice (weighing $35 \pm 2 \mathrm{~g}$ ) from GFP transgenic mice (GFP mice), for a total of nineteen mice, were used in this study (Table.1).

Table 1. Experimental Periods and Number of Specimens

\begin{tabular}{llllll}
\hline & Cont & 4 day & 7 day & 14 day & Total \\
\hline No & $5(2)$ & $5(2)$ & $5(2)$ & $5(2)$ & $20(8)$ \\
\hline (): GFP mice
\end{tabular}

GFP mice were 7-week-old female C57BL/ 6 recipient mice (Charles River) and 7-week-old female GFP transgenic mice (C57BL/6-Tg (CAG-EGFP)) (Shimizu Lab. Supplies Co., Ltd., Kyoto, Japan).

To prepare for bone marrow transplantation, GFP transgenic mice were sacrificed under general anesthesia by isoflurane inhalation, and immediately we extracted the femur and removed the soft tissue, and harvested donor bone marrow cells suspended in RPMI 1640 medium plate with anti-biotic, displacement HBBS immediately after 7-week-old female C57BL/ 6 recipient mice had undergone 10 Gy of lethal whole-body-irradiation split and $1 \times 10^{7}$ bone marrow cells were injected into the tail vein of the recipients (24-26).

The recipient GFP mice were used 5 weeks after transplantation. The mice were kept in an air-conditioned room with controlled temperature at $24 \pm 1^{\circ} \mathrm{C}$. The mice were housed in a breeding room with a 12-hour cycle of day and night and controlled in paper-lined plastic cages (Paper Clean: Peparlet Co., Ltd., Hamamatsu, Japan). The mice were freely fed with solid food (Picolab Rodent Diet 20; Japan SLC Inc., Hamamatsu, Japan) and water. The physical condition of the mice was good and there were few fluctuations in their weight during the examination.

\section{2) Experimental methods}

Each mouse was placed on a hand-made experiment table in a dorsal position under general anesthesia by intraperitoneal injection of Somnopenty $1^{\circledR}$ $40 \mathrm{mg} / \mathrm{kg}$ (Pentobarbital sodium, Kyoritsu Seiyaku Corp., Tokyo, Japan). Using a \#1/4 jet carbide bar (\#432296 1/4, Shofu Inc., Kyoto, Japan) and a straight hand-piece drill, we created a guiding hole in the occlusal surface of upper left first molar. A micro-plus-screwpin (head part: $1.7 \mathrm{~mm}$ in diameter and $0.5 \mathrm{~mm}$ thickness, Ohsato, Saitama, Japan) was screwed into the guiding hole and fixed to the tooth. The occlusal surface of the upper left first molar was raised by the $0.5 \mathrm{~mm}$ thickness of the head of the micro-plus-screwpin (Fig.1) (23).

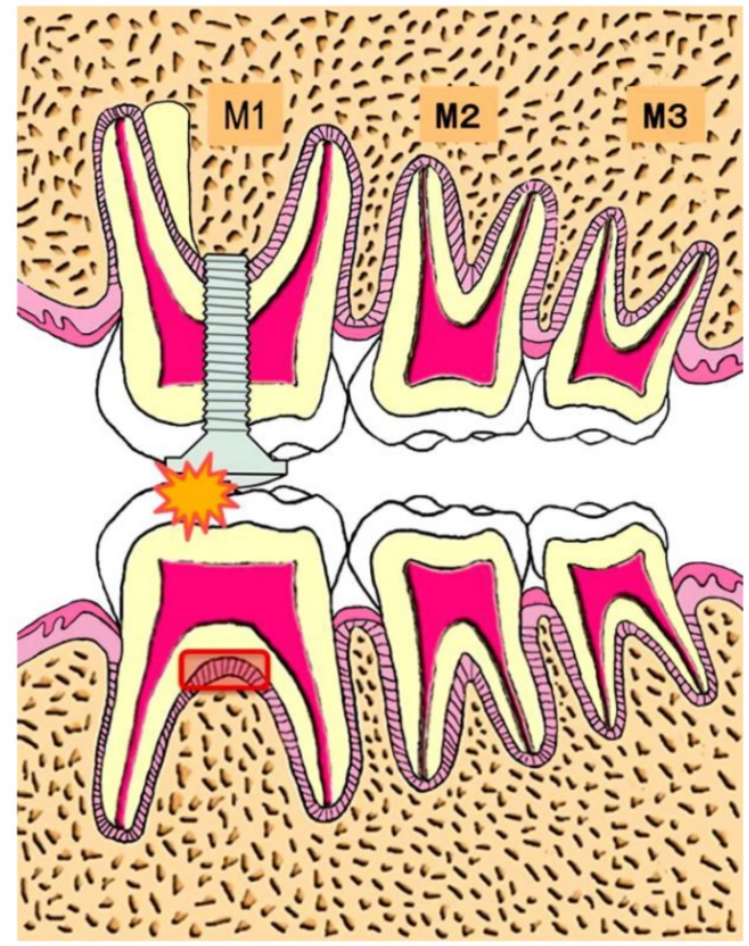

Figure 1. Experimental schema 
R_mCT was used to confirm the occlusal contact between upper left first molar and lower left first molar (23). At 4, 7, and 14 days after increasing occlusal height, the mice were sacrificed by an overdose of pentobarbital sodium. Five mice served as a control group. A total five of $\mathrm{ddY}$ and C57BL/ 6 genealogy mice served as each experimental group on days 4,7 , and 14 (Table.1). Specimens containing the furcation area of the lower-left-first molar were fixed in $10 \%$ neutral buffered formalin solution, demineralized in $10 \%$ EDTA, dehydrated in increasing series of alcohol in a routine manner and embedded in paraffin. Bucco-lingual serial sections of $4 \mu \mathrm{m}$ thickness were prepared and stained in hematoxylin-eosin. We used left first molar periodontal ligament of normal mice in the control group.

The ethics committee on laboratory animals at Matsumoto Dental University approved the examination (Number \#233-13).

\section{3) Histopathological examination}

Histopathological changes of the periodontal tissues at the furcation area of the lower left first molar and its surrounding periodontal tissues were observed under a light microscope. We noted the small change form of cell nuclei and performed digital image analysis using Adobe ${ }^{\circledR}$ Photoshop CC 2014 (Adobe Systems Software Ireland Ltd., CA, U.S.A) to confirm the number of cells of the periodontal ligament at the furcation area of the lower left first molar.

\section{4) Immunohistochemical examination}

For immunohistochemical staining, the slides were deparaffinized in xylene followed by antigen retrieval in $10 \mathrm{mM}$ citric acid buffer solution, $\mathrm{pH} 6.0$ at $121^{\circ} \mathrm{C}$ for $15 \mathrm{~min}$. This was followed by blocking with hydrogen peroxide methanol solution for $10 \mathrm{~min}$ processing, and with devitalized endogenous peroxidase.

About Ki67, the primary antibody was monoclonal Rat Anti-Mouse Ki-67Antigen. Clone TEC-3
Code No. M7249 (DakoCytomation, Denmark) with a dilution of $1: 100$, reaction overnight at $4^{\circ} \mathrm{C}$ and the secondary antibody was monoclonal mouse antibody Simple stain mouse MAX-PO(M) (Nichirei Co. Ltd., Tokyo, Japan). After washing by PBS and DAB staining, specimens were counterstained with hematoxylin. For negative control, PBS was used instead of primary antibody. We counted the number of dyed cells in the periodontal ligament of the optional area.

About GFP, the primary antibody was Anti-GFP antibody-ChlP Grade ab290 (abcam ${ }^{\circledR}$, Cambridge, UK) with 1:5000, overnight at $4^{\circ} \mathrm{C}$ and the secondary antibody was rabbit polyclonal antibody Simple stain mouse MAX-PO(R) (Nichirei, Tokyo, Japan). After washing by PBS and DAB staining development, specimens were counterstained with hematoxylin. For negative control, PBS was used instead of the primary antibody.

\section{5) Digital Image Analyze and Statistical Analyze Methods}

For semi-quantitative evaluation of histopathological at immunohistochemical staining, the following procedure was performed. First, the histopathological-photographic images with same magnification from the related examination were prepared and one pixel density was counted for each image. Then, typical staining (hematoxylin; IHC-DAB) part was defined as position area. The pixel number percentage of the positive area was compared with the total pixel number percentage of the same area, and the ratio was obtained. The statistical analysis was Mann-Whitney U Test using SPSS28). The analyzed area of the cement-enamel junction (CEJ) in bucco-lingual position of the furcation area was drawn with a straight line, and a perpendicular line was drawn from CEJ to the alveolus bone. Cell nuclei were picked out and we calculated the pixel share of area of the cell nuclei part (Fig.2). Further, we excluded a gap in a blood vessel cavity in this analyzed part.

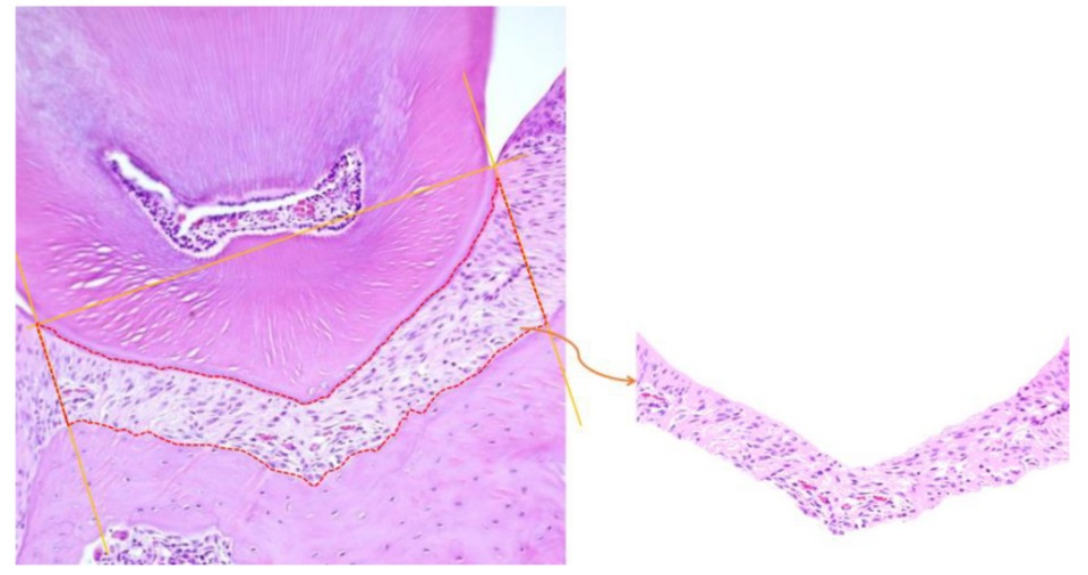

Figure 2. Histopathological photograph of the observation site 


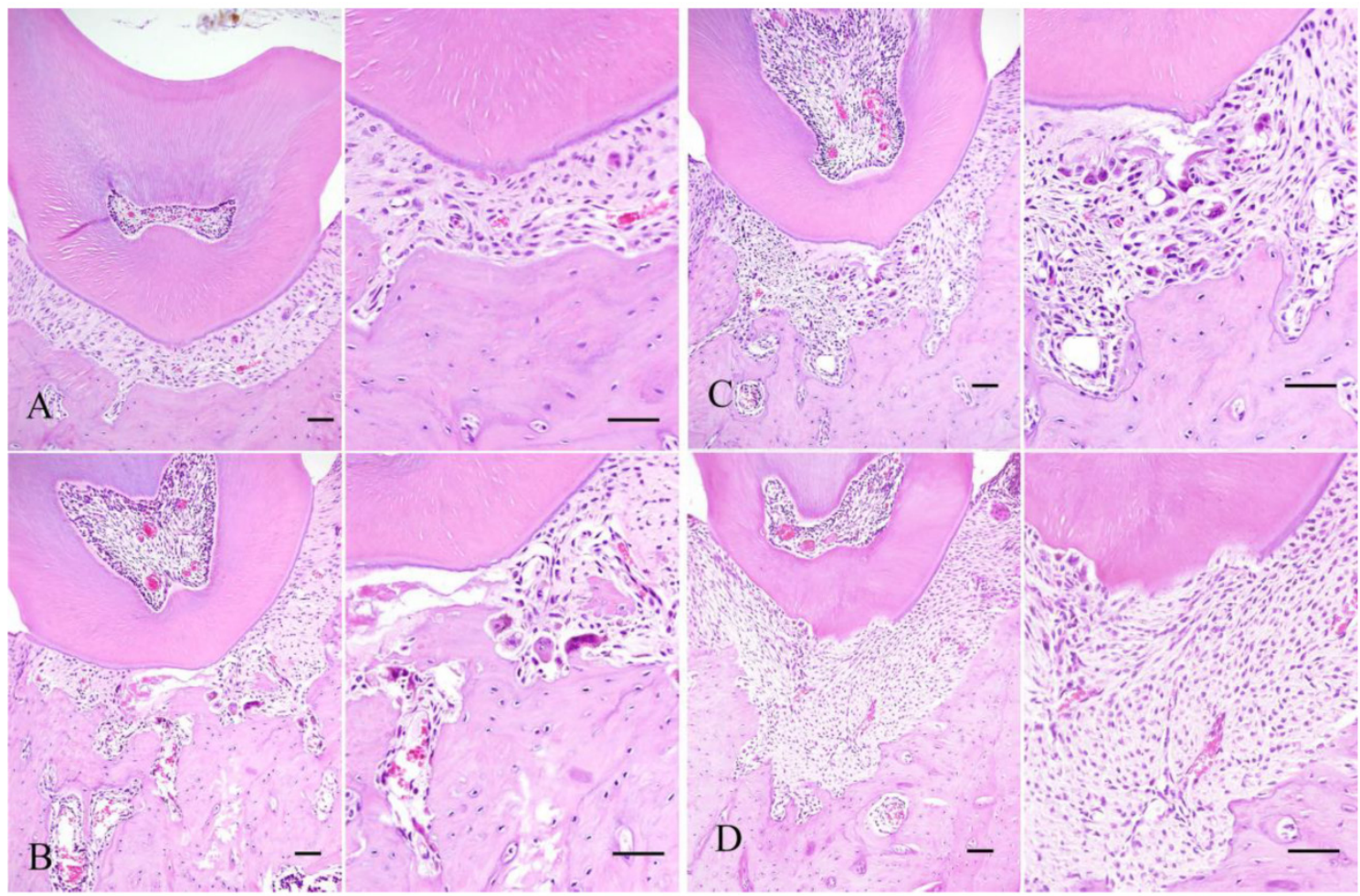

Figure 3. Histopathology of control group specimen (A), experimental 4 day group specimen (B), experimental 7 day group specimen (C) and experimental 14 day group specimen (D). Scale bar: $50 \mu \mathrm{m}$.

\section{Result}

In this experiment, the histopathological difference in the ddY mouse and the GFP mouse was not recognized. Throughout the experimental period, no inflammatory reaction was observed at the epithelium around the respective teeth.

\section{1) Histopathological examination}

In the control group, the periodontal ligament maintained a constant width, and main fibers ran across the cementum and the alveolar bone in an orderly manner. The fibroblasts in the periodontal ligament appeared spindle shaped among the collage bundles. Cell nuclei existed densely relatively, periodontal ligament fiber had the part of a minute capillary, and an erythrocyte was filled with in the blood vessel cavity. Furthermore, a cellular cementum clearly existed (Fig.3-A).

In the experimental group on day 4 , the periodontal ligament was somewhat tightly compressed and the capillary hyperemia spindle evident. The spindle cells, in which hematoxylin deeply stained round-shaped nuclei, were increased in number. Multinucleate giant cell appeared mainly on the alveolus bone surface. It absorbed the undermining bone tissue, making some lacunae (Fig.3-B).

In the experimental group on day 7 , the cells with round nuclei were decreased compared with the experimental group on day 4. Hyperemia of the blood vessels developed. The alveolus bone surface of Howship's lacunae formation displayed multinucleate giant cells at the cementum. Hyaline degeneration enlargement was also observed in the specimen from day 4. Furthermore, a cellar cementum break down was evident at the furcation of the periodontal ligament surface (Fig.3-C).

In the experimental group on day 14, the resorption area on the cementum and the alveolar bone surface accompanied with multinucleated giant cells were expanding rapidly. The cells with round nuclei decreased. The nuclei and the cytoplasm, both of which indicate the shape of the spindle, were seen again. The periodontal ligament cavity became wider (Fig.3-D).

In the cytological kinetics method, we analyzed the nuclei share of pixel to compare the all pixels of the area, using photographic the nucleic (hemotoxic-deeply-stained-portion) analysis defined in the related periodontal tissue, both in the experiment and control specimens. In the all experimental groups, the results were the following: the pixel share of related area of the control group $(6.7 \pm 1.6)$, experimental group on day $4(11.3 \pm 1.2)$, day $7(9.3 \pm 2.1)$ and day $14(9.3 \pm 1.6)$. Especially, experimental group on day 4 showed a significant increase (Scheffe Test, $\mathrm{p}<0.05$ ). Compared with experimental group on day 4, experimental group on days 7 and 14 decreased but 
mostly same degree share and they were not significant to compare with the control group (Scheffe Test, $\mathrm{p}>0.05$ ) (Fig.4).

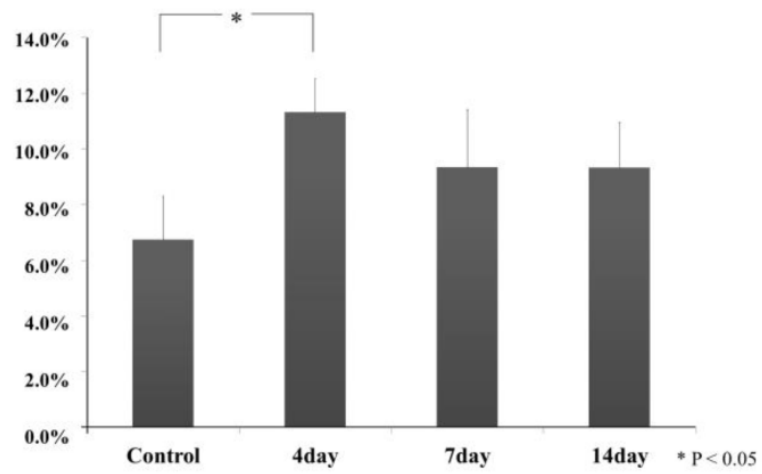

Figure 4. Hemotoxylin-deeply-stained-portion sharing ratio

\section{2) Immunohistochemical examination observations}

In control group, Ki67 positive cell was hardly seen, and it was a small round-shape in the periodontal ligament. In the experimental group on day 4 , it increased in number and deeply stained round-shape. In the experimental group on day 7 , it decreased in number but appeared spindle shaped deeply stained. In the experimental on day 14, it became fewer and was not different than the number of the control group. According the digital image analysis method, we decided on the range $(118 \times 54 \mathrm{~mm})$ and counted the number of cells in the range and then divided the number of Ki67-positive cell by the number of overall cells. The number of Ki67 positive cells in the periodontal ligament at an experimental group on day 4 (17.2 \pm 4.1$)$ had increased significantly compared with control group $(4.4 \pm 2.2)$ (Tukey Test, $\mathrm{p}<0.05)$. For the experimental group on day 7 , compared to the experimental group on day 4 , it tended to reduce but it indicated significance $(14.7 \pm 2.2)$ (Tukey Test, $\mathrm{p}<0.05)$. In the experimental group on day 14 (9.0 $\pm 3.7)$, it was not significant but it increased compared with the control group (Tukey Test, $\mathrm{p}>0.05$ ) (Fig.5,6).

In the control group, and the experimental group on day 4 and day 14, there were sparse GFP positive cells that it was stained round-shape in the periodontal ligament. According to the digital image analyze method, in the experimental group on day 7, GFP positive cells increased in number and deeply stained round-shape. GFP positive cell pixel share of periodontal ligament at the experimental group on day 7 $(19.7 \pm 6.8)$ were increased significantly larger, compared with the control group $(8.6 \pm 1.8)$ (Tukey Test, $\mathrm{p}<0.05)$. The experimental group on day $4(7.7 \pm 1.6)$ and day $14(7.6 \pm 2.7)$ was not recognized significantly (Tukey Test, $p>0.05$ ) (Fig. 7, 8).

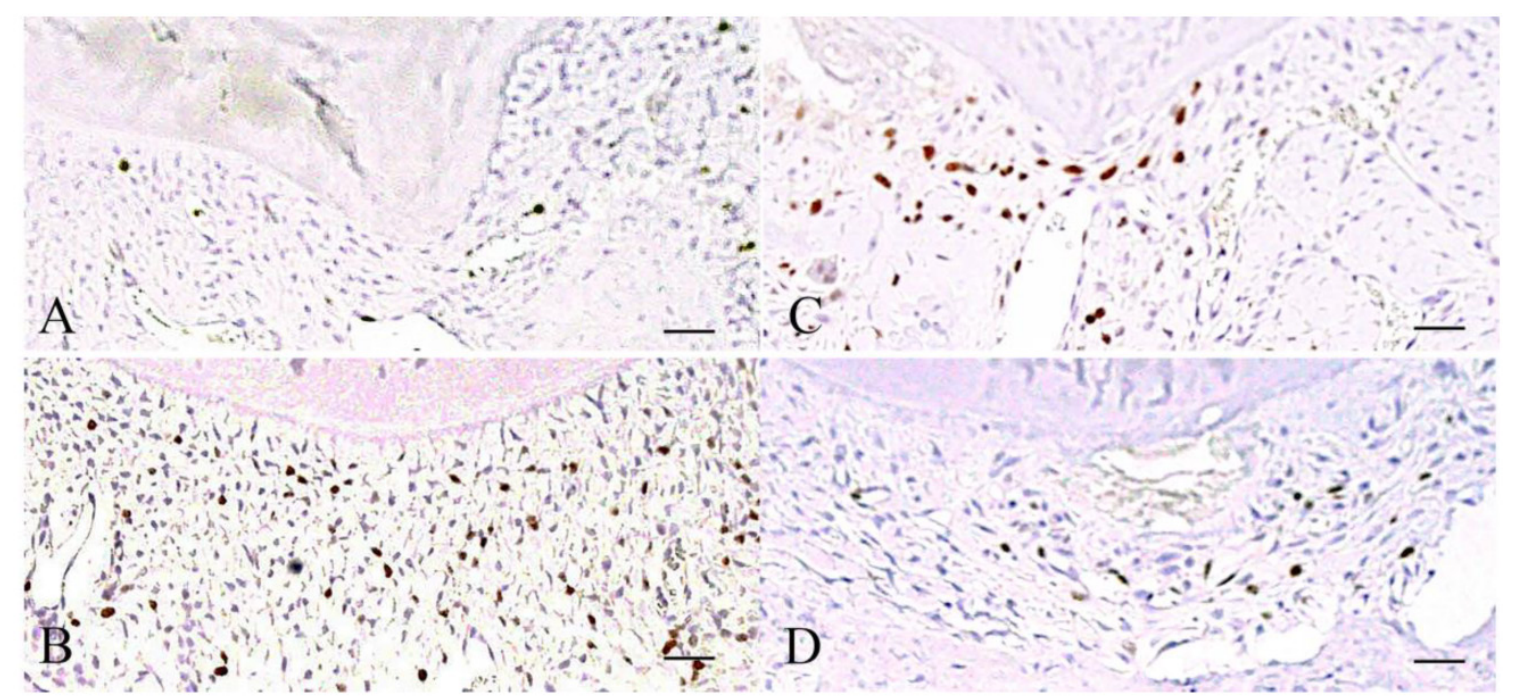

Figure 5. Ki67 IHC staining specimens. Control (A), Experimental 4 day (B), Experimental 7 day (C) and Experimental 14 day (D). Scale bar: $50 \mu m$. 


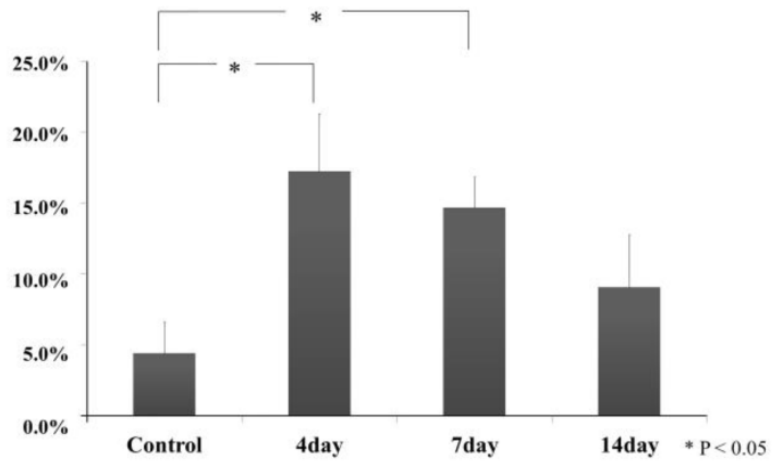

Figure 6. Ki67-positive cell ratio

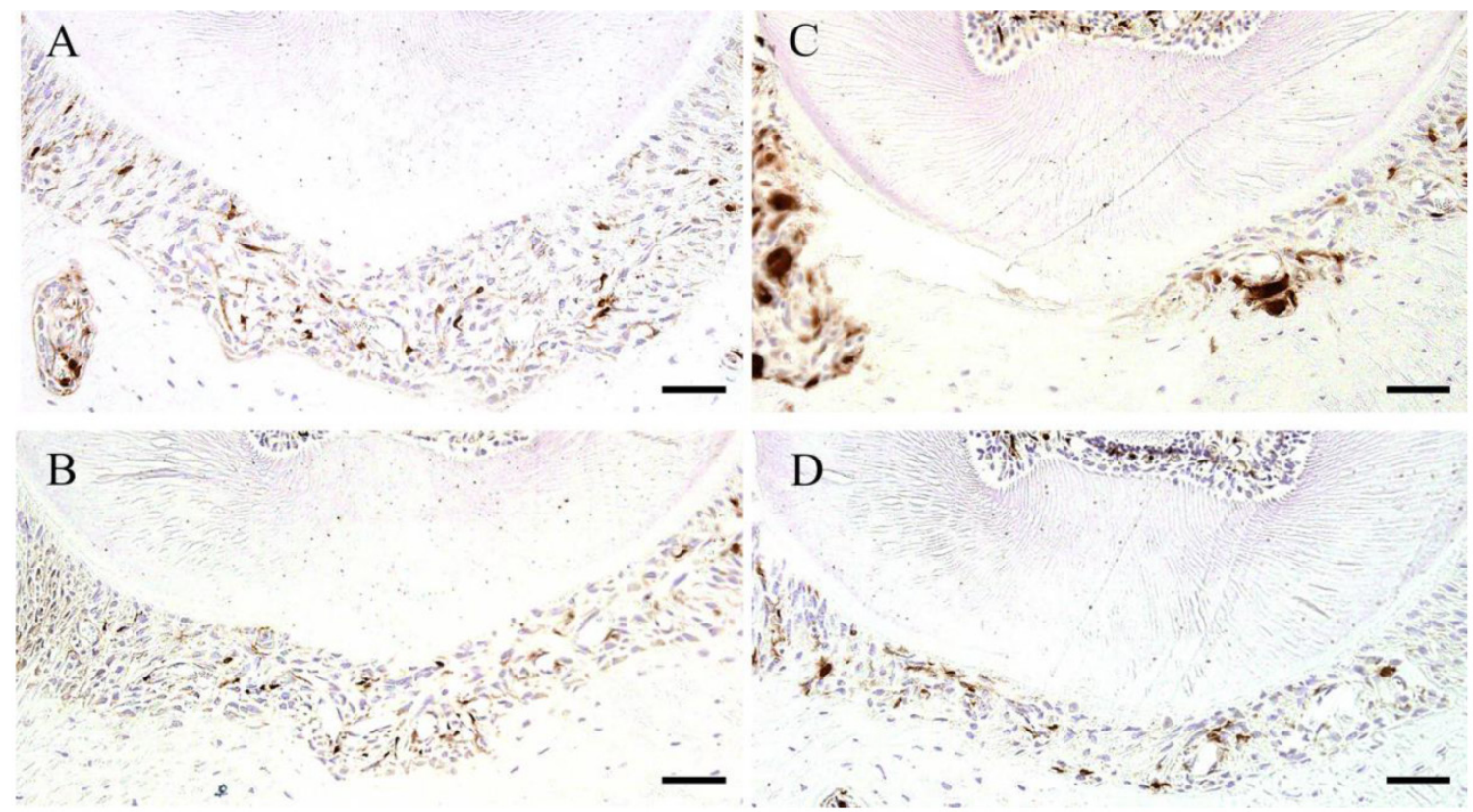

Figure 7. IHC staining of GFP. Control specimen (A), Experimental 4 day specimen (B), Experimental 7 day specimen (C) and Experimental 14 day specimen (D). Scale bar: $50 \mu \mathrm{m}$.

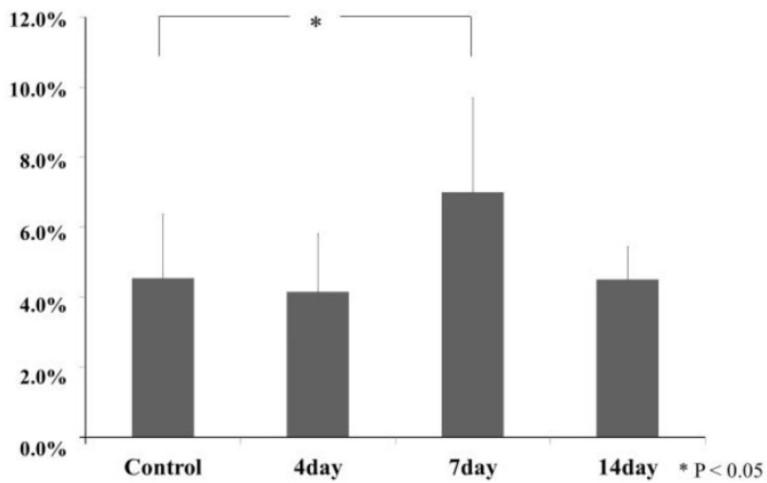

Figure 8. GFP positive area share ratio

\section{Discussion}

It is known that traumatic occlusion in the presence of periodontal tissues along with plaque-induced inflammation may have an important contributory role in the progression of periodontal disease (6). The periodontal ligament and histopathology-like consideration is mostly experimental of rats, macaque monkeys and beagle dogs as seen in animal experiments performed so far (9-11, 17-19). However, the report did not find a focus point at cytological kinetics of periodontal ligament due to excessive occlusal loading. Thus, we focused the cytological kinetics in the periodontal tissues by excessive occlusal loading.

In several studies, an occlusal trauma experimental model has been carried out using various animals. Experimental in vivo models by the way of wrought crowns, casting inlays, or orthodontic square wires attached to the maxillary posterior teeth with mathylmethacrylate resin, which lead to the bite-up and occlusal trauma for the related teeth $(9-11,17-20)$.

In this study, we established an experimental model of occlusal trauma in mice, and analyzed histopathological and immunohistochemical changes of 
cytological kinetics in periodontal ligament of the lower left first molar (Fujii-MS).

At first, we discuss the usefulness of the experimental model used in the examination. The common clinical evidence of occlusal trauma teeth is vibration and destabilization of the teeth, and the vibration at the time of occlusion and the destabilization of teeth at the time of grinding mean that periodontal tissue is burdened with an excessive force. Therefore, without using the complicated definition of "jiggling force", we used mice to determine a change of the periodontal tissue due to an occlusal trauma by observing the vibration of the tooth at the time of the excessive occlusal loading. Wentz et al. (21) explained that "jiggling force" was an external force shaking a tooth which was generated because the tooth crown was burdened by a force from one direction in a certain instant, and subsequently from the opposite direction in next instant, and these movements were repeated. As for a mandibular movement cycle in mice, it is relatively simple. It has little grinding, and an experiment system can be simplified by burdening it with bite pressure under occlusal stress in the tooth axis direction, and it is easy to analyze because dyeing methods are highly diversified. In this study, the experiment system with reproducibility in mice was established. Mice also have other advantages such as easy breeding and many types of staining. It is possible to set the high state of the tooth uniformly by implanting a micro-plus-screwpin with a head high diameter of a unified standard to the occlusal surface of maxillary first molar of the mouse, and to reduce the possibility of detachment during the experiment period by using tightening torque. Thus, the establishment of a useful experimental model on an extremely small animal, in which the loading is both at a high enough level and is intermittent, is indispensable and allows detailed investigation of periodontal tissues reaction on mechanical loading (23).

Next, we discuss the histopathological and immunohistochemical examination results. Histopathologicaly, the periodontal ligament at the furcation area of the molar teeth in the experimental group on day 4 the hyperemia of the blood vessels and increasing of cell periodontal ligament at furcation area of molar teeth were evident. In the experimental group on day 7 , it caused hyaline degeneration. It developed on occlusal trauma by excessive occlusal loading having been strongly added to periodontal ligament in the examination.

The previous study has investigated the bonding of a fixed steel wire to the upper first molar occlusal surface without inflammatory reaction and observed the histopathological change and width of the periodontal ligament state of the molar tooth area of excessive occlusal loading the periodontal ligament at days 3, 5, 7 and 14 (20). Although it is different during the experiment periods of the histopathological change by the experimental animals, the histopathological changes were similar to our results. We believe this experimental model was useful for analysis of occlusal trauma pathogenesis.

The periodontal ligament increased significantly in the experimental group on day 4, and thus the cell density was rising. This phenomenon suggests that the periodontal ligament cells increased, and it was thought that the tissues have recovery potential. After that an excessive occlusal loading which continue to the periodontal ligament caused a hyaline degeneration and some other damage, and the possibility of cell extinction was suggested. In the experimental group on day 7 , the nuclei pixel share of periodontal ligament recognized the tendency of reduction. In experimental group on day 14, there were no significant differences between the experimental and control specimens, thus periodontal ligament suggested adaptation against excessive occlusal loading. The Ki67 positive cells in periodontal ligament of the experimental group on day 4 were a value of approximately two times than that of control group. Ki67 is a related nucleoprotein in the cell cycle. Therefore, it means that cell division activity existed in periodontal ligament at the furcation area of the tooth which received an injury in the experimental group on day 4 , and it can be guessed that it is going to participate in restoration.

From observation of GFP-staining specimens, it significantly increases highly in the experimental group on day 7 , but in the experimental group on day 14 there was no significant difference in comparison with the control group. All the cells constituting each tissue of GFP transgenic mouse express Green Fluorescent Protein. No matter what kind of cell the transplanted bone marrow-derived cell differentiates into, it is traceable owing to GFP. It is reported in the result of previous experiments that a lot of GFP positive cells migrated to the periodontal tissues of the mouse after a marrow transplant and such cell are also identified osteoclast and a macrophage (16). In addition, there are also a dendritic cell and periodontal ligament fibroblast as the cell at which movement and cell differentiation are estimated in the periodontal tissues.

In this experiment, the bone marrow-derived cell on the periodontal ligament at the furcation area of occlusal trauma increased with GFP mouse in the experiment group on day 7 . It is suggested that the GFP-positive main cell is an osteoclast and a macrophage by previous study. It cannot adapt to only with the periodontal ligament cell at the injury part by 
continuous excessive occlusal force pressure, so it is thought that it induces a remodeling to need the mobilization of the bone marrow-derived cell.

According to the study, the continuative remodeling of periodontal ligament with the acceleration of the cell activation is induced from the experimental group on day 4 in the injury part of periodontal ligament at the furcation area by occlusal trauma of excessive occlusal loading. From the experimental group on day 7 , it suggests that remodeling of mechanism is recognized significantly by osteoclast and macrophage from bone marrow-derived cell for the injury part of periodontal ligament at the furcation area. Thus, when there was no or slight inflammation of gingivitis, the periodontal ligament of occlusal trauma suggested that it could expect organization adaptation by the cell of the periodontal ligament and bone marrow-derived cells.

\section{Acknowledgment}

This study was supported by Grants-in-Aid for Scientific Research \# 26463104 and \# 25463204 from the Japan Society for the Promotion of Science. The authors thank Professor DM Carlson for his reading of the manuscript.

\section{Author Contributions}

T. Takaya and H. Mimura contributed to conception, mouse examination, staining of $\mathrm{H}-\mathrm{E}$ and IHC, design, data acquisition, analysis interpretation, and drafted the manuscript and final version of the manuscript; S. Matsuda, contributed to analyzed R_mCT; K. Nakano, contributed to conception, staining of H-E and IHC; H. Tsujigiwa contributed to conception, GFP mouse; M. Tomida, contributed to analyzed data acquisition; N. Okafuji and T. Fujii, contributed to conception, and interpretation; T. Kawakami contributed to conception, and supervision, and he critically revised the manuscript. All authors gave final approval and agree to be accountable for all aspects of the work.

\section{Competing Interests}

The authors have declared that no competing interest exists.

\section{References}

1. Svanberg G. Influence of trauma from occlusion on the periodontium of dogs with normal or inflamed gingivae. Odontol Revy. 1974; 25: 165-178.

2. Stahl SS. Accommodation of the periodontium to occlusal trauma and inflammatory periodontal disease. Dent Clin North Am 1975; 19: 531-542.

3. Lindhe J, Ericsson I. The influence of trauma from occlusion on reduced but healthy periodontal tissues in dogs. J Clin Periodontol. 1976; 3: 110-122.

4. Biancu S, Ericsson I, Lindhe J. Periodontal ligament tissue reactions to trauma and gingival inflammation. An experimental study in the beagle dog. J Clin Periodontol. 1995; 22: 772-779.

5. Glickman I, Smulow JB. Effect of excessive occlusal forces upon the pathway of gingival inflammation in humans. J Periodontol. 1965; 36: 141-147.
6. Waerhaug J. The Infrabony Pocket and its Relationship to Trauma from Occlusion and Subgingival plaque. J Periodontol. 1979; 7: 355-365.

7. Polson AM. The relative importance of plaque and occlusion in periodontal disease. J Clin Periodontol. 1986; 13: 923-927.

8. Harrel SK. Occlusal forces as a risk factor for periodontal disease. Periodontol 2000. 2003; 32: 111-117.

9. Lindhe J, Svanberg G. Influence of trauma from occlusion on progression of experimental periodontitis in the beagle dog. J Clin Periodontol. 1974; 1: 3-14.

10. Ericsson I, Lindhe J. The effect of elimination of jiggling forces on periodontally exposed teeth in the dog. J Periodontol. 1982, 53: 562-567.

11. Svanberg G, Lindhe J. Vascular reactions in the periodontal ligament incident to trauma from occlusion. J Clin Periodontol. 1974; 1: 58-69.

12. Liang JiaBi, Hiroshi Kato. Periodontal Destruction with Experimental Periodontitis and Occlusal Trauma in Mokeys. J Periodontol. 1996; 38(4): 385-399.

13. Okafuji N, Nakano K, Yamaki T, et al. Experimentally Traumatic Mechanical Stress exposed Periodontal Tissues. J.J. A.D.T. 2011; 7(1): 25-31.

14. Wall DA, Hamberg SD, Reynolds DS, et al. Immunodeficiency in graft-versus-host disease. I. Mechanism of immune suppression. J Immunol. 1998; 40: 2970-2976.

15. Zijlmans JM, Visser JW, Laterveer L, et al. The early phase of engraftment after murine blood cell transplantation is mediated by hematopoietic stem cells. Proc Natl Acad Sci USA. 1998; 95: 725-729.

16. Muraoka M, Tsujigiwa H, Nakano K, et al. Transplanted Bone Marrow-derived cell Migration into Periodontal Tissues and Cell Differentiation. J. Hard Tissue Biol. 2011; 20: 301-306.

17. Budtz-Jørgensen E. Occlusal dysfunction and stress. An experimental study in macaque monkeys. J Oral Rehabil. 1981; 8: 1-9.

18. Zhang G, Huang $X$ and Herring SW. Effect of unilateral bite splint on mastication in the miniature pig. J Oral Rehabil. 1994; 21: 613-622.

19. Sodeyama T, Maeda T, Takano Y, Hara M. Responses of periodontal nerve terminals to experimentally induced occlusal trauma in rat molars: an immunohistochemical study using PGP 9.5 antibody. J Periodontal Res. 1996; 31: 235-248.

20. Kaku M, Uoshima K, Yamashita Y, et al. Investigation of periodontal ligament reaction upon excessive occlusal load - osteopontin induction among periodontal ligament cells. J Periodont Res. 2005; 40: 59-66.

21. FM Wentz, J Jarabak, B Orban. Experimental occlusal trauma imitating cuspal interferences. J Periodontol. 1958; 29: 117-127

22. Okayasu I, Yamada Y, Kohno S, et al. New Animal Model for Studying Mastication in Oral Motor Disorders. J Dent Res. 2003; 82(4): 318-321.

23. Fujii T, Takaya T, Mimura H, et al. Experimental Model of Occlusal Trauama in Mouse Periodontal Tissues. J. Hard Tissue Biol. 2014; 23(3): 377-380.

24. Yuan Y-W, Tamamura R, Lei L, et al. The Ability of Transplanted Bone Marrow-Derived Cells to Differentiate into Parenchymal Cells of Salivary Glands. J Hard Tissue Biology. 2013; 22(4):433-438.

25. Noda Y, Nishizaki K, Yoshinobu J, et al. The engraftment and differentiation of transplanted bone marrow-derived cells in the olfactory bulb after methimazole administration. Acta Otolaryngol. 2013; 133(9):951-956.

26. Tsujigiwa H, Hirata Y, Katase N, et al. The Role of Bone Marrow-Derived Cells During the Bone Healing Process in the GFP Mouse Bone Marrow Transplantation Model. Calcif Tissue Int. 2013; 92(3):296-306.

27. Tomida M, Tsujigiwa H, Nakano K, et al. Promotion of Transplanted Bone Marrow-derived Cell Migration into the Periodontal Tissues due to Orthodontic Mechanical Stress. Int J Med Sci. 2013; 10(10):1321-1326. 